University of Nebraska - Lincoln

DigitalCommons@University of Nebraska - Lincoln

Agronomy \& Horticulture -- Faculty Publications

Agronomy and Horticulture Department

2004

\title{
Emergence and Survival of Legumes Seeded into Pastures Varying in Landscape Position
}

John A. Guretzky

University of Nebraska-Lincoln, jguretzky2@unl.edu

Kenneth J. Moore

lowa State University, kjmoore@iastate.edu

Allen D. Knapp

lowa State University

E. Charles Brummer

lowa State University

Follow this and additional works at: https://digitalcommons.unl.edu/agronomyfacpub

Part of the Plant Sciences Commons

Guretzky, John A.; Moore, Kenneth J.; Knapp, Allen D.; and Brummer, E. Charles, "Emergence and Survival of Legumes Seeded into Pastures Varying in Landscape Position" (2004). Agronomy \& Horticulture -Faculty Publications. 543.

https://digitalcommons.unl.edu/agronomyfacpub/543

This Article is brought to you for free and open access by the Agronomy and Horticulture Department at DigitalCommons@University of Nebraska - Lincoln. It has been accepted for inclusion in Agronomy \& Horticulture -Faculty Publications by an authorized administrator of DigitalCommons@University of Nebraska - Lincoln. 


\title{
Emergence and Survival of Legumes Seeded into Pastures Varying in Landscape Position
}

\author{
John A. Guretzky, ${ }^{*}$ Kenneth J. Moore, Allen D. Knapp, and E. Charles Brummer
}

\begin{abstract}
Landscape position affects legume establishment in pastures. We conducted this study to (i) determine the role of emergence and survival on establishment of legumes on summit and backslope positions and (ii) examine how competition, as influenced by sward-cutting height and $\mathbf{N}$ fertilization, affects legume emergence and survival across these positions. We no-till drilled a mixture composed of red clover (Trifolium pratense L.), alfalfa (Medicago sativa $\mathbf{L}$.), and birdsfoot trefoil (Lotus corniculatus L.) into an existing cool-season grass pasture at Rhodes, IA, in 1998 and 1999. Treatments consisted of landscape positions (summit and backslope), $\mathbf{N}$ fertilizer rates $(0,22$, 44 , and $89 \mathrm{~kg} \mathrm{ha}^{-1}$ ), and sward heights (uncut, 5 , and $13 \mathrm{~cm}$ ). Landscape position did not affect legume emergence in swards cut at 5 and 13 cm. At those heights, emergence averaged 1041 and 831 seedlings $\mathrm{m}^{-2}$. In swards cut at $5 \mathrm{~cm}, 62$ plants $\mathrm{m}^{-2}$ survived on summits vs. 183 plants $\mathrm{m}^{-2}$ on backslopes. Legume emergence and survival generally decreased as sward height and $\mathbf{N}$ fertilization increased but interactions with landscape position occurred. Addition of $89 \mathrm{~kg} \mathrm{ha}^{-1} \mathrm{~N}$ was required to reduce survival on backslopes to 39 plants $\mathrm{m}^{-2}$, a density similar to that on summits. Our results show that seedling survival limits legume establishment on summit positions in pastures. Legume establishment is successful on backslopes because of less competition from grass. We recommend that legumes be seeded on backslope positions in pastures, $\mathbf{N}$ fertilizer not be applied, and grass competition be reduced before seeding legumes in pastures.
\end{abstract}

TEUMEs improve the production and nutritional value of forage in cool-season grass pastures. Pastures composed of grasses and legumes produce more dry matter than grass-only pastures (Taylor and Allinson, 1983). Through symbiotic $\mathrm{N}_{2}$ fixation with rhizobial bacteria, legumes increase soil mineral N (Heichel, 1985). The transfer of $\mathrm{N}$, from the decomposition of roots and leaf residues of legumes, improves the nutritional value and growth of the grass (Heichel and Henjum, 1991). Legumes also are higher in crude protein than are grasses (Van Soest, 1982), and their establishment with cool-season grasses can improve the seasonal distribution of forage growth and quality in pastures (Sleugh et al., 2000).

Harmoney et al. (2001) found establishment of legumes to vary according to landscape position. Legumes contributed more to forage production on backslope than on summit or toeslope positions, and the proportion of dry matter comprised of legumes was related linearly to diversity of leguminous species in pastures. They could not conclude why legume establishment differed at summit and backslope landscape positions, al-

Dep. of Agronomy, Iowa State Univ., Ames, IA 50011-1010. Received 1 July 2002. *Corresponding author (john.a.guretzky@erdc.usace. army.mil).

Published in Crop Sci. 44:227-233 (2004).

(c) Crop Science Society of America

677 S. Segoe Rd., Madison, WI 53711 USA though differential competitive pressures from established grasses may have been the cause.

Competition from grass often limits the establishment of legumes in pastures. Legume interseeding is most successful when competition from grasses is reduced through herbicide application (Cuomo et al., 2001; Groya and Sheaffer, 1981) or by cutting swards to low heights before seeding legumes (Taylor and Allinson, 1983). Soil N availability also influences grass-legume competition. Grasses have a greater relative growth rate than legumes in areas with high soil $\mathrm{N}$ availability. In areas with low soil $\mathrm{N}$ availability, legumes are more competitive because they can supplement mineral $\mathrm{N}$ uptake with $\mathrm{N}_{2}$ fixation (Schwinning and Parsons, 1996).

How competition, as influenced by sward height and $\mathrm{N}$ fertilization, affects legume establishment in pastures varying in landscape position is unknown. Our objectives were to (i) determine the role of emergence and survival during the seeding year on establishment of several legumes sown as a mixture on summit and backslope positions in pastures and (ii) examine how sward height and $\mathrm{N}$ fertilization affect emergence and survival of legumes on these landscape positions.

\section{MATERIALS AND METHODS}

This experiment was conducted at the Iowa State University Rhodes Research Farm ( $41^{\circ} 52^{\prime}$ N, $93^{\circ} 10^{\prime}$ W). A 1737 pure live seed (PLS) $\mathrm{m}^{-2}$ mixture consisting of red clover (T. pratense 'Marathon'), alfalfa ( $M$. sativa 'Alfagraze'), and birdsfoot trefoil (L. corniculatus 'Norcen') was no-till drilled using a cone seeder (Almaco, Nevada, IA) into a cool-season grass pasture composed of three cool-season grasses: smooth brome (Bromus inermis Leyss.), Kentucky bluegrass (Poa pratensis L.), and reed canarygrass (Phalaris arundinacea $\mathrm{L}$.). The legumes were sown at $582 \mathrm{PLS} \mathrm{m}^{-2}$ of red clover, $587 \mathrm{PLS} \mathrm{m}^{-2}$ of alfalfa, and $568 \mathrm{PLS} \mathrm{m}^{-2}$ of birdsfoot trefoil. On a PLS basis, these rates corresponded to $11.3 \mathrm{~kg} \mathrm{ha}^{-1}$ red clover, $12.6 \mathrm{~kg}$ $\mathrm{ha}^{-1}$ alfalfa, and $8.6 \mathrm{~kg} \mathrm{ha}^{-1}$ birdsfoot trefoil. The legumes were inoculated with a humus-based rhizobium specific to each legume.

We conducted the experiment in small plots, $1.4 \mathrm{~m}^{2}$ in 1998 and $2.8 \mathrm{~m}^{2}$ in 1999 , to facilitate counts of legume emergence and survival under several treatments. Treatments consisted of two landscape positions (summit and backslope), four $\mathrm{N}$ fertility rates $\left(0,22,44\right.$, and $\left.89 \mathrm{~kg} \mathrm{ha}^{-1}\right)$ applied as $\mathrm{NH}_{4} \mathrm{NO}_{3}$, and three sward heights (uncut, 5 , and $13 \mathrm{~cm}$ above the soil). The backslope had a west-facing aspect and 10\% slope. The summit had zero aspect and slope. Tillage and herbicide burndown are common procedures to establish legumes in pastures. However, we attempted to follow procedures of Harmoney et al. (2001), reducing competition from existing grasses by a mechanical rather than a chemical means. Also, because aspect does not affect legume establishment or persistence in

Abbreviations: LAI, leaf area index; PLS, pure live seed. 
these pastures (Harmoney et al., 2001), it was not incorporated into this experiment.

For several years before 1998, the pasture was fertilized annually in the spring with $\mathrm{N}, \mathrm{P}$, and $\mathrm{K}$ at 77,26 , and $34 \mathrm{~kg}$ $\mathrm{ha}^{-1}$, respectively, cut once for hay, and grazed by cattle July through August. In 1998, ground adjacent to the experimental plots was cut and raked with a tractor but was not fertilized or grazed. Plots in 1999 were arranged $\approx 3 \mathrm{~m}$ away from the 1998 plots. Before seeding, the abundance of litter appeared to be similar among 1998 and 1999 plots.

Plots were seeded on 28 Apr. 1998 and 20 May 1999. Nitrogen treatments were applied on 27 Apr. 1998 and 26 Apr. 1999. Wet-field conditions delayed planting following $\mathrm{N}$ application in 1999. Cutting dates in 1998 were 27 April, 7 May, 19 May, and 19 June for the 5-cm height treatment and 7 May, 19 May, and 19 June for the 13-cm height treatment. In 1999, the cutting dates were 26 Apr., 21 May, and 7 June for the 5-cm height and 21 May and 7 June for the 13-cm height. Uncut swards were not cut until August of each year. In August, all plots were cut at $13 \mathrm{~cm}$. The 5 and $13-\mathrm{cm}$ height treatments consisted of clipping the grass with a sickle bar push mower and lightly hand-raking the plots. Mowing did not result in a chopping of the grass, only clipping at the respective height. Raking removed the fresh, clipped grass lying on top of the canopy but did not disturb existing litter. Clipping was terminated before legume seedlings reached $5 \mathrm{~cm}$ in height.

Our goal with the sward height and $\mathrm{N}$ fertilizer rate treatments was to establish several levels of competition. Because sward height treatments were used only to reduce competition from existing grasses, sward heights or yields of grass were not measured at the time of clipping. We clipped the $5-\mathrm{cm}$ height treatment frequently to inhibit grass competition. The $13-\mathrm{cm}$ height treatment was cut less frequently and the uncut sward not at all to establish the different levels of competition.
Because of wet field conditions, the 5 and $13-\mathrm{cm}$ height treatments were cut one less time in 1999 than in 1998.

We measured legume emergence using a destructive sampling technique. The technique involved counting, identifying, and then removing each legume seedling that emerged within a $0.1-\mathrm{m}^{2}$ subplot. Emergence was measured on 15 May, 4 June, and 30 June 1998 and 9 and 28 June 1999. As a percentage of overall emergence, 88,10 , and $2 \%$ of legumes emerged in 1998 and 80 and $20 \%$ of overall legumes emerged in 1999 by those dates, respectively. The data we present represents the sum of emergence after all sampling dates each year. Seedling survival was measured within a different $0.1-\mathrm{m}^{2}$ subplot on 15 Aug. 1998 and 1999, 109 and 87 d after planting in 1998 and 1999, respectively. We used a LAI-2000 Plant Canopy Analyzer (LI-COR, Lincoln, NE) to measure leaf area index (LAI) of the grass swards. Leaf area index was measured on 28 May and 22 June 1998 and 22 June and 16 July 1999, before the legumes produced appreciable leaf area.

We conducted the experiment in a randomized complete block. Four replicated blocks were established on each landscape position, and separate blocks were established for each seeding year. Within each block, $\mathrm{N}$ fertilizer rate and swardheight treatments were arranged in a split plot. Nitrogen fertilizer rate treatments were randomly arranged as whole plots. Sward height treatments were randomly arranged within each $\mathrm{N}$ fertilizer rate as subplots. Every block consisted of 12 plots. We considered the effects of landscape position, $\mathrm{N}$, and sward height fixed and replications random. We conducted an analysis of variance to test these effects by using $F$ ratios for splitplot experiments arranged in a randomized complete block design (McIntosh, 1983). Because interactions of treatments and years occurred, the analysis of variance was conducted by year (Table 1 ). Significance was defined at $P \leq 0.05$. However, if treatment effects were significant at $P \leq 0.10$ in one

Table 1. Analysis of variance (ANOVA) for emergence and survival of a legume mixture interseeded into a cool-season grass pasture at Rhodes, IA, in 1998 and 1999. Sources of variation included two landscape positions (L), four $\mathbf{N}$ fertilizer rates (N), three sward heights (H), and four replications (R). Mean squares (MS) are presented for overall legume, red clover, alfalfa, and birdsfoot trefoil emergence and survival by year.

\begin{tabular}{|c|c|c|c|c|c|c|c|c|c|c|}
\hline \multirow[b]{2}{*}{ Source } & \multirow[b]{2}{*}{$F$ test } & \multirow[b]{2}{*}{ df } & \multicolumn{2}{|c|}{ Overall legume } & \multicolumn{2}{|c|}{ Red clover } & \multicolumn{2}{|c|}{ Alfalfa } & \multicolumn{2}{|c|}{ Birdsfoot trefoil } \\
\hline & & & 1998 MS & 1999 MS & 1998 MS & 1999 MS & 1998 MS & 1999 MS & 1998 MS & 1999 MS \\
\hline & & & \multicolumn{8}{|c|}{ Emergence } \\
\hline $\mathbf{L}$ & $\operatorname{MS}(\mathbf{L}) / \mathbf{M S}[\mathbf{R}(\mathbf{L})]$ & 1 & 12558 & 349451 & 33153 & 76784 & 1240 & 33153 & 66993 & 17281 \\
\hline $\mathbf{R}(\mathbf{L})$ & & 63 & 342513 & 168992 & 51142 & 32288 & 51369 & 24841 & 31961 & 10162 \\
\hline $\mathbf{N}$ & $\mathbf{M S}(\mathbf{N}) / \mathbf{M S}[\mathbf{R} \times \mathbf{N}(\mathbf{L})]$ & 3 & 70243 & 1921573 **** & 19460 & $338338 * * *$ & 7327 & $202810 * * *$ & 24648 & $128961 * * *$ \\
\hline $\mathbf{L} \times \mathbf{N}$ & $\mathbf{M S}(\mathbf{L} \times \mathbf{N}) / \mathbf{M S}[\mathbf{R} \times \mathbf{N}(\mathbf{L})]$ & 3 & 46980 & 251941 & 11246 & 54633 & 16379 & 23298 & 9960 & 18608 \\
\hline $\mathbf{R} \times \mathbf{N}(\mathbf{L})$ & & 181 & 133481 & 170476 & 23891 & 32362 & 20545 & 20435 & 11726 & 15793 \\
\hline $\mathbf{H}$ & $\mathbf{M S}(\mathbf{H}) / \mathbf{M S}[\mathbf{R} \times \mathbf{N} \times \mathbf{H}(\mathbf{L})]$ & 21 & 164054 & $3774791 * * *$ & 10578 & 784986 **** & 4026 & $287179 * * *$ & $74123 * *$ & $278910 * * *$ \\
\hline $\mathbf{L} \times \mathbf{H}$ & $\mathbf{M S}(\mathbf{L} \times \mathbf{H}) / \mathbf{M S}[\mathbf{R} \times \mathbf{N} \times \mathbf{H}(\mathbf{L})]$ & 21 & 104999 & 139936 & 17186 & 35253 & 17078 & 7707 & 16028 & 10166 \\
\hline $\mathbf{N} \times \mathbf{H}$ & $\mathbf{M S}(\mathbf{N} \times \mathbf{H}) / \mathbf{M S}[\mathbf{R} \times \mathbf{N} \times \mathbf{H}(\mathbf{L})]$ & 61 & $196746 *$ & 119832 & 18517 & 23271 & $33996 * *$ & 9208 & 20323 & 12468 \\
\hline $\mathbf{L} \times \mathbf{N} \times \mathbf{H}$ & $\mathbf{M S}(\mathbf{L} \times \mathbf{N} \times \mathbf{H}) / \mathbf{M S}[\mathbf{R} \times \mathbf{N} \times \mathbf{H}(\mathbf{L})]$ & 62 & $243468 * *$ & $237022 \dagger$ & $44423 * *$ & $62937 *$ & $25312 \dagger$ & 21462 & $21741 \dagger$ & 15384 \\
\hline \multirow[t]{2}{*}{$\mathbf{R} \times \mathbf{N} \times \mathbf{H}(\mathbf{L})$} & & 36 & 69957 & 113257 & 14490 & 24133 & 11299 & 14577 & 11359 & 32989 \\
\hline & & & \multicolumn{8}{|c|}{ Survival } \\
\hline $\mathbf{L}$ & $\operatorname{MS}(\mathbf{L}) / \mathbf{M S}[\mathbf{R}(\mathbf{L})]$ & 1 & $61560 * *$ & 173740 **** & $45067 *$ & $66993 * * *$ & 13 & $1935 \dagger$ & $1528 \dagger$ & $13090 * *$ \\
\hline $\mathbf{R}(\mathbf{L})$ & & 6 & 4111 & 1193 & 2101 & 686 & 108 & 425 & 284 & 493 \\
\hline $\mathbf{N}$ & $\mathbf{M S}(\mathbf{N}) / \mathbf{M S}[\mathbf{R} \times \mathbf{N}(\mathbf{L})]$ & 3 & 5552 & $25370 * * *$ & 2967 & $5387 *$ & 82 & $1015 *$ & 570 & $3684 * *$ \\
\hline $\mathbf{L} \times \mathbf{N}$ & $\mathbf{M S}(\mathbf{L} \times \mathbf{N}) / \mathbf{M S}[\mathbf{R} \times \mathbf{N}(\mathbf{L})]$ & $\mathbf{3}$ & 4911 & $9435 *$ & 2859 & 2257 & 13 & 387 & 573 & 1359 \\
\hline $\mathbf{R} \times \mathbf{N}(\mathbf{L})$ & & 18 & 3998 & 2839 & 2652 & 1075 & 56 & 249 & 303 & 615 \\
\hline $\mathbf{H}$ & $\mathbf{M S}(\mathbf{H}) / \mathbf{M S}[\mathbf{R} \times \mathbf{N} \times \mathbf{H}(\mathbf{L})]$ & 2 & $51145 * * *$ & $161044 * * *$ & $28574 * * *$ & $46014 * * *$ & $\mathbf{8 5 5}$ *** & $7770 * * *$ & $1423 * * *$ & $10076 * * *$ \\
\hline $\mathbf{L} \times \mathbf{H}$ & $\mathbf{M S}(\mathbf{L} \times \mathbf{H}) / \mathbf{M S}[\mathbf{R} \times \mathbf{N} \times \mathbf{H}(\mathbf{L})]$ & 2 & $3911 \dagger$ & $50809 * * *$ & $3609 *$ & $18095^{* * * *}$ & 41 & $922 \dagger$ & 129 & $3771 * * *$ \\
\hline $\mathbf{N} \times \mathbf{H}$ & $\mathbf{M S}(\mathbf{N} \times \mathbf{H}) / \mathbf{M S}[\mathbf{R} \times \mathbf{N} \times \mathbf{H}(\mathbf{L})]$ & 6 & 2215 & $11677 *$ & 1495 & $3604 *$ & 70 & 467 & 170 & $1729 * * *$ \\
\hline $\mathbf{L} \times \mathbf{N} \times \mathbf{H}$ & $\mathbf{M S}(\mathbf{L} \times \mathbf{N} \times \mathbf{H}) / \mathbf{M S}[\mathbf{R} \times \mathbf{N} \times \mathbf{H}(\mathbf{L})]$ & 6 & 1415 & 7784 & 700 & 2578 & 18 & 217 & 290 & $1104 * *$ \\
\hline $\mathbf{R} \times \mathbf{N} \times \mathbf{H}(\mathbf{L})$ & & 36 & 1568 & 4262 & 828 & 1583 & 56 & 374 & 233 & 369 \\
\hline
\end{tabular}

* Denotes significance at the 0.05 probability level.

** Denotes significance at the 0.01 probability level.

*** Denotes significance at the $\mathbf{0 . 0 0 1}$ probability level.

$\dagger$ Denotes significance at the 0.100 probability level. 
year and $P \leq 0.05$ the other year, both were considered significant.

\section{RESULTS \\ Emergence}

\section{Landscape Position Effects}

In 1998 and 1999, overall legume emergence was similar among summits and backslopes at all $\mathrm{N}$ rates in swards cut at 5 and $13 \mathrm{~cm}$ (Table 2). At those heights, overall legume emergence averaged 1041 and 831 seedlings $\mathrm{m}^{-2}$ across years. Overall legume emergence was greater on backslopes than on summits by 686 plants $\mathrm{m}^{-2}$ in uncut swards fertilized at $22 \mathrm{~kg} \mathrm{ha}^{-1} \mathrm{~N}$ in 1998 and 1003 plants $\mathrm{m}^{-2}$ in uncut swards at $0 \mathrm{~kg} \mathrm{ha}^{-1} \mathrm{~N}$ in 1999. Emergence was greater for red clover than alfalfa and birdsfoot trefoil in 1998 and 1999. Landscape position effects on emergence of each species, however, were similar to their effects on overall legume emergence (Table 2).

\section{Nitrogen Fertilization Effects}

Nitrogen fertilization did not affect overall legume emergence in swards cut at $5 \mathrm{~cm}$ in 1998 or 1999 nor in swards cut at $13 \mathrm{~cm}$ in 1998 (Table 2). Addition of $\mathrm{N}$ from 0 to $89 \mathrm{~kg} \mathrm{ha}^{-1}$ reduced overall legume emergence by $68 \%$ in swards cut at $13 \mathrm{~cm}$ in 1999 . In uncut swards, addition of $\mathrm{N}$ from 0 to $89 \mathrm{~kg} \mathrm{ha}^{-1}$ reduced overall legume emergence on backslopes by 37\% in 1998 and $92 \%$ in 1999. Nitrogen fertilization had negligible effects on overall legume emergence on summits. Effects of $\mathrm{N}$ fertilization on red clover, alfalfa, and birdsfoot trefoil emergence were similar to those on overall legume emergence in 1998 and 1999.

\section{Sward Height Effects}

Overall legume emergence was similar among sward heights at most $\mathrm{N}$ fertilization rates on summits and backslopes in 1998. In 1999, increased sward height reduced overall legume emergence at all $\mathrm{N}$ rates on summits and at 44 and $89 \mathrm{~kg} \mathrm{ha}^{-1} \mathrm{~N}$ on backslopes by 61 to $98 \%$ (Table 2). Sward height affected the emergence of red clover, alfalfa, and birdsfoot trefoil similarly as overall emergence in 1998 and 1999.

\section{Survival}

\section{Landscape Position Effects}

Landscape position and sward height interactions affected overall legume survival in 1998 and 1999 (Table 1). In swards cut at $5 \mathrm{~cm}$, overall legume survival was greater on backslopes than on summits by 69 plants $\mathrm{m}^{-2}$ in 1998 and 173 plants $\mathrm{m}^{-2}$ in 1999 (Table 3). In swards cut at $13 \mathrm{~cm}$ and in uncut swards, overall legume survival was, on average across years, greater on backslopes than on summits by 60 and 22 plants $\mathrm{m}^{-2}$, respec-

Table 2. Landscape position, sward height, and $\mathbf{N}$ fertilizer rate effects on emergence of legumes interseeded into a cool-season grass pasture at Rhodes, IA. Legumes were interseeded as a mixture across summit (S) and backslope (B) landscape positions on 28 Apr. 1998 and 20 May 1999. Values represent sum of overall legume, red clover, alfalfa, and birdsfoot trefoil emergence after measuring on 15 May, 4 June, and 30 June 1998 and 9 and 28 June $1999(n=4)$.

\begin{tabular}{|c|c|c|c|c|c|c|c|c|c|c|c|}
\hline \multirow[b]{4}{*}{ Legume species } & \multirow[b]{4}{*}{ Year } & \multirow[b]{4}{*}{ Sward height } & \multicolumn{9}{|c|}{ Emergence of legumes } \\
\hline & & & \multicolumn{8}{|c|}{ Nitrogen, $\mathbf{k g ~ h a}^{-1}$} & \multirow[b]{3}{*}{ SE } \\
\hline & & & \multicolumn{2}{|c|}{$\mathbf{0}$} & \multicolumn{2}{|c|}{22} & \multicolumn{2}{|c|}{44} & \multicolumn{2}{|c|}{89} & \\
\hline & & & $\mathbf{S}$ & B & $\mathbf{S}$ & B & $\mathbf{S}$ & B & $\mathbf{S}$ & $\mathbf{B}$ & \\
\hline & & cm & & & 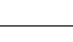 & - seed & $\mathbf{m}^{-2}$ & & & & \\
\hline \multirow[t]{6}{*}{ Overall } & 1998 & 5 & 1047 & 853 & 893 & 842 & 1025 & 1028 & 1103 & 1023 & $233.0 \dagger$ \\
\hline & & 13 & 675 & 1052 & 893 & 648 & 807 & 950 & 971 & 710 & 213.5 \\
\hline & & Uncut & 1068 & 1068 & 678 & 1364 & 960 & 686 & 500 & 673 & $187.0 \S$ \\
\hline & 1999 & 5 & 1217 & 1480 & 1386 & 1015 & 958 & 969 & 818 & 993 & 257.1 \\
\hline & & 13 & 1074 & 1066 & 928 & 1168 & 791 & 880 & 361 & 329 & 257.2 \\
\hline & & Uncut & 345 & 1348 & 412 & 608 & 374 & 162 & 14 & 108 & 238.0 \\
\hline \multirow[t]{6}{*}{ Red clover } & 1998 & 5 & 441 & 288 & 385 & 315 & 498 & 374 & 412 & 406 & 119.6 \\
\hline & & 13 & 315 & 398 & 388 & 266 & 355 & 409 & 422 & 302 & 114.8 \\
\hline & & Uncut & 481 & 398 & 291 & 508 & 525 & 323 & 245 & 323 & 117.4 \\
\hline & 1999 & 5 & 506 & 684 & 654 & 420 & 490 & 466 & 371 & 493 & 115.9 \\
\hline & & 13 & 544 & 474 & 428 & 525 & 369 & 428 & 180 & 202 & 115.9 \\
\hline & & Uncut & 159 & 614 & 197 & 288 & 178 & 102 & 3 & 62 & 109.8 \\
\hline \multirow[t]{6}{*}{ Alfalfa } & 1998 & 5 & 371 & 248 & 280 & 315 & 334 & 328 & 390 & 307 & 113.4 \\
\hline & & 13 & 213 & 363 & 312 & 258 & 296 & 377 & 355 & 261 & 107.6 \\
\hline & & Uncut & 361 & 339 & 288 & 516 & 261 & 229 & 202 & 207 & 110.5 \\
\hline & 1999 & 5 & 415 & 436 & 415 & 342 & 256 & 304 & 240 & 280 & 91.9 \\
\hline & & 13 & 266 & 345 & 261 & 350 & 243 & 245 & 108 & 65 & 90.9 \\
\hline & & Uncut & 135 & 401 & 129 & 237 & 170 & 49 & 11 & 41 & 85.3 \\
\hline \multirow[t]{6}{*}{ Birdsfoot trefoil } & 1998 & 5 & 234 & 318 & 229 & 213 & 194 & 326 & 301 & 309 & 81.1 \\
\hline & & 13 & 148 & 291 & 194 & 124 & 156 & 164 & 194 & 148 & 75.8 \\
\hline & & Uncut & 226 & 331 & 100 & 339 & 175 & 135 & 54 & 143 & 75.4 \\
\hline & 1999 & 5 & 296 & 361 & 318 & 253 & 213 & 199 & 207 & 221 & 75.0 \\
\hline & & 13 & 264 & 248 & 240 & 294 & 181 & 207 & 73 & 62 & 76.5 \\
\hline & & Uncut & 51 & 334 & 86 & 83 & 27 & 11 & 0 & 6 & 69.6 \\
\hline
\end{tabular}

$+\mathrm{SE}$, by year and legume species, for comparison of two landscape position means at the same or different $\mathrm{N}$ rate and sward height.

$+\mathrm{SE}$, by year and legume species, for comparison of two $\mathrm{N}$ rate means at the same landscape position and sward height.

$\S \mathrm{SE}$, by year and legume species, for comparison of two sward height means at the same $\mathrm{N}$ rate and landscape position. 
Table 3. Landscape position and sward height effects on survival of legumes interseeded as a mixture into a cool-season grass pasture at Rhodes, IA. Values represent overall legume, red clover, alfalfa, and birdsfoot trefoil survival on 15 Aug. 1998 and 1999 ( $n=16)$.

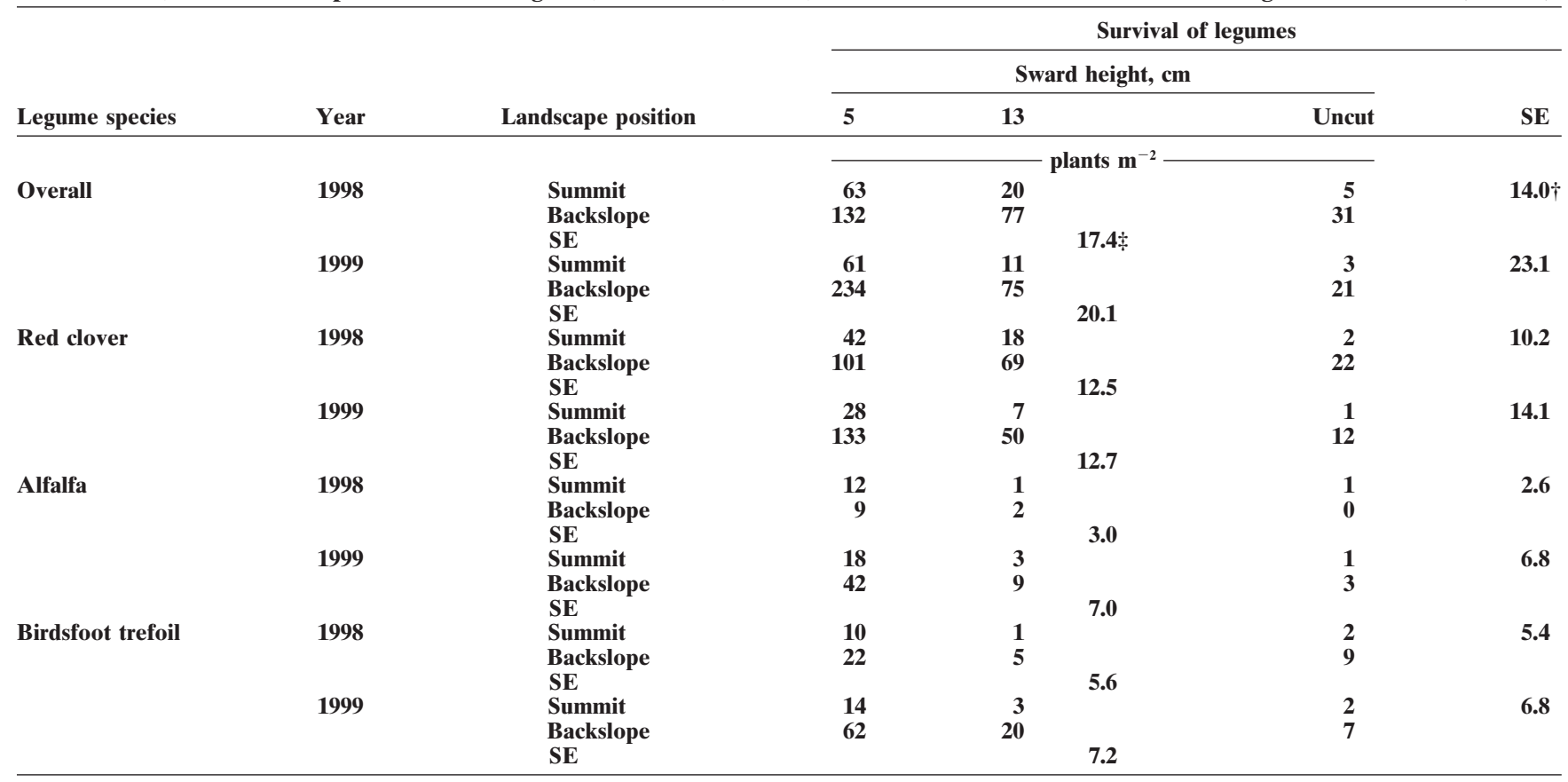

† SE by year and legume species for comparison of two sward height means at the same landscape position.

+ SE by year and legume species for comparison of two landscape position means at the same or different sward height.

tively (Table 3 ). Red clover had the greatest survival of the legume species and was affected similarly by landscape position as overall legume survival in 1998 and 1999.

Survival of alfalfa and birdsfoot trefoil was similar among summits and backslopes in 1998 (Table 3). In 1999, alfalfa survival was 24 plants $\mathrm{m}^{-2}$ greater on backslopes than on summits in plots cut at $5 \mathrm{~cm}$ but similar among landscape positions in swards cut at $13 \mathrm{~cm}$ and in uncut swards. Birdsfoot trefoil survival was also greater on backslopes than on summits in plots cut at $5 \mathrm{~cm}$ (Table 3) and in unfertilized swards cut at $13 \mathrm{~cm}$ in 1999 (data not shown).

\section{Nitrogen Fertilization Effects}

Overall legume survival was also affected by a landscape position and $\mathrm{N}$ fertilization interaction in 1999. Neither $\mathrm{N}$ main effects nor the landscape position and $\mathrm{N}$ interaction on overall legume survival were statistically significant in 1998 (Table 1). In both years, addition of $\mathrm{N}$ from 0 to $44 \mathrm{~kg} \mathrm{ha}^{-1}$ reduced overall legume survival similarly on summits and backslopes (Fig. 1). Across these rates, overall legume survival was greater on backslopes than on summits by 64 plants $\mathrm{m}^{-2}$ in 1998 and 105 plants $\mathrm{m}^{-2}$ in 1999. When $\mathrm{N}$ fertilization increased to $89 \mathrm{~kg} \mathrm{ha}^{-1}$, overall legume survival declined on backslopes to 39 plants $\mathrm{m}^{-2}$ across years, a density similar to that on summits (Fig. 1).

The effect of $\mathrm{N}$ fertility on overall legume survival also varied with sward height in 1999. In swards cut at $5 \mathrm{~cm}$, overall legume survival increased from 155 plants $\mathrm{m}^{-2}$ at $0 \mathrm{~kg} \mathrm{ha}^{-1} \mathrm{~N}$ to 217 plants $\mathrm{m}^{-2}$ at $22 \mathrm{~kg} \mathrm{ha}^{-1} \mathrm{~N}$ (Table 4). In 13-cm and uncut swards, addition of $\mathrm{N}$ fertilizer decreased overall legume survival. Similar results occurred for red clover and birdsfoot trefoil as overall legume survival in 1999. Survival of alfalfa decreased with increased $\mathrm{N}$ fertilization at all sward heights in 1999. Nitrogen fertilization did not affect red clover, alfalfa, or birdsfoot trefoil survival in 1998 (Table 1).

\section{Sward Height Effects}

Overall legume survival was greatest in swards cut at $5 \mathrm{~cm}$ (Table 3). Across years, overall legume survival on summits was 46 and 58 plants $\mathrm{m}^{-2}$ greater in swards cut at $5 \mathrm{~cm}$ than in swards cut at $13 \mathrm{~cm}$ and in uncut swards, respectively. On backslopes in 1998 and 1999, respectively, overall legume survival was greater in swards cut at $5 \mathrm{~cm}$ than swards cut at $13 \mathrm{~cm}$ by 55 and 159 plants $\mathrm{m}^{-2}$ and greater in swards cut at $5 \mathrm{~cm}$ than uncut swards by 101 and 213 plants $\mathrm{m}^{-2}$. Individually, survival of each legume species also decreased with increased sward height in 1998 and 1999 (Table 3).

\section{Sward Leaf Area Index}

Leaf area index of the grass sward increased at a greater rate on summits than on backslopes. From 1 to 22 June 1998, LAI increased from 3.0 to 4.7 on summits and from 2.3 to 2.9 on backslopes (Table 5). Similar results occurred between 22 June and 16 July 1999. Leaf area index also increased with addition of $\mathrm{N}$ fertilizer (Table 5). Landscape position and $\mathrm{N}$ fertilizer rate interactions did not occur, however. Cutting at a greater height also increased LAI of the grass sward in 1998 and 1999 (Table 5). 
Table 4. Sward height and $\mathbf{N}$ fertilizer rate effects on survival of legumes interseeded as a mixture into a cool-season grass pasture at Rhodes, IA. Values represent overall legume, red clover, alfalfa, and birdsfoot trefoil survival on 15 Aug. 1998 and 1999 ( $n=8$ ).

\begin{tabular}{|c|c|c|c|c|c|c|c|}
\hline \multirow[b]{3}{*}{ Legume species } & \multirow[b]{3}{*}{ Year } & \multirow[b]{3}{*}{ Sward height } & \multicolumn{5}{|c|}{ Survival of legumes } \\
\hline & & & \multicolumn{4}{|c|}{ Nitrogen, $\mathbf{k g ~ h a ~}^{-1}$} & \multirow[b]{2}{*}{ SE } \\
\hline & & & $\mathbf{0}$ & 22 & 44 & 89 & \\
\hline & & $\mathbf{c m}$ & $\longrightarrow$ & 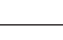 & 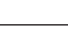 & - & \\
\hline \multirow[t]{8}{*}{ Overall } & 1998 & 5 & 126 & 100 & 79 & 85 & $24.3 \dagger$ \\
\hline & & 13 & 58 & 44 & 71 & 22 & \\
\hline & & Uncut & 30 & 40 & 4 & $\mathbf{0}$ & \\
\hline & & & & & & & \\
\hline & 1999 & 5 & 155 & 217 & 152 & 66 & 30.8 \\
\hline & & 13 & 107 & 26 & 38 & 2 & \\
\hline & & Uncut & 33 & 10 & 1 & 3 & \\
\hline & & SE & & & & & \\
\hline \multirow[t]{8}{*}{ Red clover } & 1998 & 5 & 90 & 67 & 65 & 65 & 18.9 \\
\hline & & 13 & 53 & 36 & 68 & 19 & \\
\hline & & Uncut & 22 & 23 & $\mathbf{3}$ & 0 & \\
\hline & & SE & & & & & \\
\hline & 1999 & 5 & 70 & 115 & 93 & 45 & 18.8 \\
\hline & & 13 & 61 & 20 & 30 & 2 & \\
\hline & & Uncut & 18 & 5 & 1 & 3 & \\
\hline & & SE & & & & & \\
\hline \multirow[t]{8}{*}{ Alfalfa } & 1998 & 5 & 11 & 16 & 4 & 10 & 3.7 \\
\hline & & 13 & 2 & 1 & 1 & 1 & \\
\hline & & Uncut & 3 & $\mathbf{0}$ & $\mathbf{0}$ & $\mathbf{0}$ & \\
\hline & & SE & & & & & \\
\hline & 1999 & 5 & 44 & 33 & 32 & 8 & 9.1 \\
\hline & & 13 & 9 & $\mathbf{1}$ & 3 & 0 & \\
\hline & & Uncut & 3 & 1 & $\mathbf{0}$ & $\mathbf{0}$ & \\
\hline & & SE & & & & & \\
\hline \multirow[t]{8}{*}{ Birdsfoot trefoil } & 1998 & 5 & 25 & 17 & 11 & 11 & 8.0 \\
\hline & & 13 & 3 & 7 & 1 & 1 & \\
\hline & & Uncut & 5 & 17 & 1 & 0 & \\
\hline & & SE & & & & & \\
\hline & 1999 & 5 & 42 & 69 & 28 & 14 & 10.6 \\
\hline & & 13 & 38 & 6 & 4 & 0 & \\
\hline & & Uncut & 12 & 5 & 1 & 1 & \\
\hline & & SE & & & & & \\
\hline
\end{tabular}

$\dagger$ SE by year and legume species for comparison of two $\mathbf{N}$ fertilizer rate means at the same or different sward height.

$¥ \mathrm{SE}$ by year and legume species for comparison of two sward height means at the same $\mathbf{N}$ fertilizer rate.

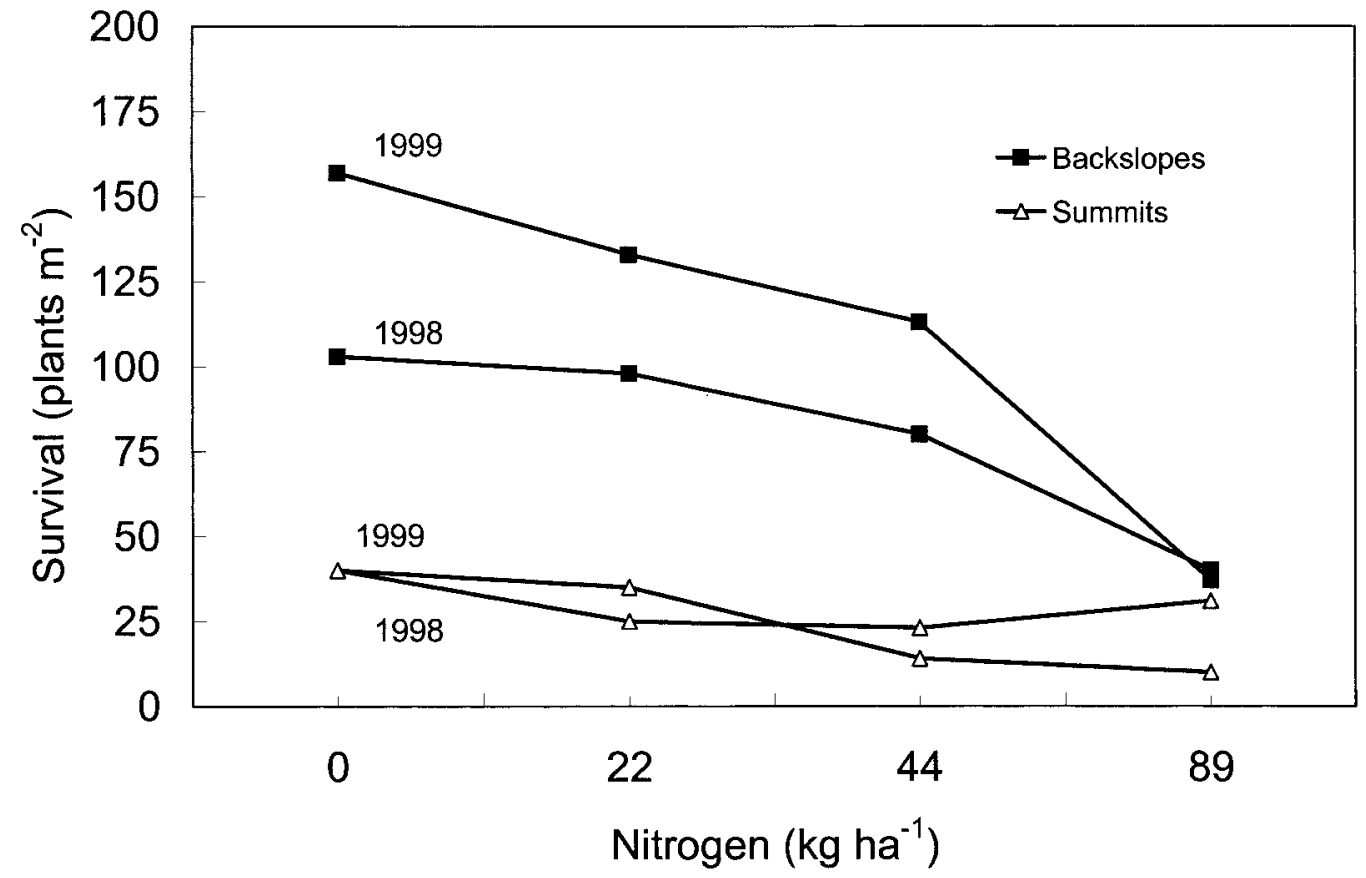

Fig. 1. Landscape position and $\mathbf{N}$ fertilizer rate effects on survival of a legume mixture interseeded into a cool-season grass pasture at Rhodes, IA. Values represent overall survival of red clover, alfalfa, and birdsfoot trefoil on 15 Aug. 1998 and 1999. Standard errors for comparison of landscape position means at the same or different $N$ rate were 25.9 in 1998 and 20.1 in 1999. Standard errors for comparison of $N$ rate means at the same landscape position were 25.8 in 1998 and 21.8 in 1999. 
Table 5. Analysis of variance and means for leaf area index of pasture swards measured at Rhodes, IA, in 1998 and 1999 . Sources of variation included two landscape positions $(L)$, four $\mathbf{N}$ fertilizer rates $(\mathbf{N})$, three sward heights $(H)$, and four replications $(R)$. Leaf area index was measured in grass swards at two sampling dates each year before accumulation of appreciable leaf area by legume seedlings.

\begin{tabular}{|c|c|c|c|c|c|c|}
\hline \multirow[b]{3}{*}{ Source } & \multirow[b]{3}{*}{$F$ test } & \multirow[b]{3}{*}{ df } & \multicolumn{4}{|c|}{ Leaf area index } \\
\hline & & & \multicolumn{2}{|c|}{1998} & \multicolumn{2}{|c|}{1999} \\
\hline & & & 28 May & 22 June & 22 June & 16 July \\
\hline $\mathbf{L}$ & $\operatorname{MS}(\mathbf{L}) / \operatorname{MS}[R(L)]$ & 1 & 11.9 & $75.7 * * *$ & 2.1 & $12.2 * *$ \\
\hline $\mathbf{R}(\mathbf{L})$ & & 6 & 3.4 & 1.8 & 1.5 & 0.5 \\
\hline $\mathbf{N}$ & $\mathbf{M S}(\mathbf{N}) / \mathbf{M S}[\mathbf{R} \times \mathbf{N}(\mathbf{L})]$ & 3 & $5.4 * * *$ & $2.1 \dagger$ & $3.9 * *$ & $9.2 * * *$ \\
\hline $\mathbf{L} \times \mathbf{N}$ & $\mathbf{M S}(\mathbf{L} \times \mathbf{N}) / \mathbf{M S}[\mathbf{R} \times \mathbf{N}(\mathbf{L})]$ & 3 & 0.1 & 0.2 & 1.4 & 0.7 \\
\hline $\mathbf{R} \times \mathbf{N}(\mathbf{L})$ & & 18 & 0.6 & 0.7 & 0.6 & 0.3 \\
\hline H & $\mathbf{M S}(\mathbf{H}) / \mathbf{M S}[\mathbf{R} \times \mathbf{N} \times \mathbf{H}(\mathbf{L})]$ & 2 & 43.8*** & $114.1 * * *$ & $114.9 * * *$ & $91.2 * * *$ \\
\hline $\mathbf{L} \times \mathbf{H}$ & $\mathbf{M S}(\mathbf{L} \times \mathbf{H}) / \mathbf{M S}[\mathbf{R} \times \mathbf{N} \times \mathbf{H}(\mathbf{L})]$ & 2 & 0.5 & $2.1 \dagger$ & 1.8 & 1.3 \\
\hline $\mathbf{N} \times \mathbf{H}$ & $\mathbf{M S}(\mathbf{N} \times \mathbf{H}) / \mathbf{M S}[\mathbf{R} \times \mathbf{N} \times \mathbf{H}(\mathbf{L})]$ & 6 & 0.7 & 0.5 & 0.8 & 0.8 \\
\hline $\mathbf{L} \times \mathbf{N} \times \mathbf{H}$ & $\mathbf{M S}(\mathbf{L} \times \mathbf{N} \times \mathbf{H}) / \mathbf{M S}[\mathbf{R} \times \mathbf{N} \times \mathbf{H}(\mathbf{L})]$ & 6 & 0.6 & 0.2 & $\mathbf{1 . 8} \dagger$ & 0.7 \\
\hline $\mathbf{R} \times \mathbf{N} \times \mathbf{H}(\mathbf{L})$ & & 36 & 0.4 & 0.7 & 0.8 & 0.9 \\
\hline \multirow[t]{3}{*}{ Landscape position } & Summit & & 3.0 & 4.7 & 2.8 & 4.0 \\
\hline & Backslope & & 2.3 & 2.9 & 2.5 & 3.3 \\
\hline & SE $\neq$ & & 0.38 & 0.27 & 0.25 & 0.14 \\
\hline \multirow[t]{4}{*}{ Sward height, cm } & 5 & & 1.6 & 2.0 & 0.9 & 2.2 \\
\hline & 13 & & 2.5 & 3.5 & 2.4 & 3.3 \\
\hline & Uncut & & 3.9 & 5.8 & 4.6 & 5.5 \\
\hline & SE $\$$ & & 0.16 & 0.21 & 0.22 & 0.24 \\
\hline \multirow{5}{*}{ Nitrogen, $\mathbf{k g ~ h a}^{-1}$} & 0 & & 2.2 & 3.4 & 2.2 & 2.9 \\
\hline & 22 & & 2.4 & 3.7 & 2.4 & 3.4 \\
\hline & 44 & & 3.0 & 4.0 & 2.9 & 4.0 \\
\hline & 89 & & 3.2 & 4.0 & 3.0 & 4.3 \\
\hline & SEI & & 0.22 & 0.34 & 0.22 & 0.16 \\
\hline
\end{tabular}

** Denotes significance at the 0.01 probability level.

*** Denotes significance at the 0.001 probability level.

$\uparrow$ Denotes significance at the 0.100 probability level.

† SE for comparison of landscape position means by measurement date.

$\S \mathrm{SE}$ for comparison of sward height means by measurement date.

II $\mathrm{SE}$ for comparison of $\mathrm{N}$ fertilizer rate means by measurement date.

\section{DISCUSSION}

Seedling survival rather than emergence limits establishment of legumes on summit positions in pastures. Repeated defoliation of the swards to $5 \mathrm{~cm}$ improved legume survival on summits; however, relative to backslopes, survival on summits remained low. In swards cut at $5 \mathrm{~cm}$, overall legume survival on summits was less than on backslopes by 69 plants $\mathrm{m}^{-2}$ in 1998 and 173 plants $\mathrm{m}^{-2}$ in 1999 (Table 3). Overall legume survival was greater on backslopes than on summits in swards cut at 5 and $13 \mathrm{~cm}$ despite similar legume emergence among these positions at these sward heights. Only when swards remained uncut was emergence limited on summits relative to backslopes. Emergence on summits was less than on backslopes by 686 plants $\mathrm{m}^{-2}$ in uncut swards fertilized at $22 \mathrm{~kg} \mathrm{ha}^{-1} \mathrm{~N}$ in 1998 and 1003 plants $\mathrm{m}^{-2}$ in uncut swards at $0 \mathrm{~kg} \mathrm{ha}^{-1} \mathrm{~N}$ in 1999 (Table 2). Our results support those of Harmoney et al. (2001), who found forage mass of legumes in pastures was 179 $\mathrm{kg} \mathrm{ha}^{-1}$ greater on backslopes than on summits.

Reduced grass competition improved legume survival on backslope positions. In 1998 and 1999, respectively, 132 and 234 plants $\mathrm{m}^{-2}$ survived in swards cut at $5 \mathrm{~cm}$ compared with 21 and 31 plants $\mathrm{m}^{-2}$ in uncut swards (Table 3). By repeatedly cutting the sward to 5- and $13-\mathrm{cm}$ heights, grass LAI was reduced throughout June and July, months during which legume seedlings were early in development (Table 5). Greater leaf area from grasses limits establishment of legumes in pastures by limiting the photosynthetic photon flux (Trott et al., 1988) and altering the ratio of red to far-red wavelengths
(Ballaré et al., 1990) that reaches seedlings at ground level.

Overall legume survival may have been greater among plots cut at $5 \mathrm{~cm}$ on backslopes in 1999 than in 1998 because of reduced soil fertility and competition from existing grasses. We did not measure soil nutrient status, but as mentioned previously, the pasture in which this experiment was established was fertilized and moderately grazed before 1998. During 1998, however, the sod adjacent to the plots was mowed and raked but not fertilized. In 1999, the plots were established $\approx 3 \mathrm{~m}$ away from the 1998 plots. The abundance of litter before seeding was probably similar among 1998 and 1999 plots; however, soil fertility levels may have been lower in 1999 plots. Under conditions of reduced competition and soil fertility, addition of $\mathrm{N}$ at $22 \mathrm{~kg} \mathrm{ha}^{-1}$ also may have positively benefited legume survival within the $5-\mathrm{cm}$ cut plots without increasing grass competition or diminishing nodule formation in 1999 (Table 4).

Reducing competition from grass before and after seeding of legumes is necessary to support legume emergence if planting is delayed following $\mathrm{N}$ fertilization. Overall legume emergence was not affected by sward height in 1998 but was reduced by 61 to $98 \%$ in swards left uncut compared with swards cut at $5 \mathrm{~cm}$ in 1999 (Table 2). Wet field conditions delayed planting until $30 \mathrm{~d}$ after $\mathrm{N}$ application in 1999. Within uncut swards and swards fertilized at 44 to $89 \mathrm{~kg} \mathrm{ha}^{-1} \mathrm{~N}$, light availability most likely limited legume emergence. Increased etiolation of legume seedlings as $\mathrm{N}$ fertilization rates increased under $13-\mathrm{cm}$ and uncut swards was observed. 
Overall legume survival, however, did not appear to be affected by the delay in planting as survival was similar among uncut swards and swards fertilized at 44 to 89 $\mathrm{kg} \mathrm{ha}^{-1} \mathrm{~N}$ in 1998 and 1999.

Nitrogen fertilization further escalated competition from grass, and the negative effect on legume establishment was greater when sward heights were not minimized. Nitrogen fertilization is known to favor grasses over legumes and is not a recommended practice to maintain legumes in pastures (Heichel, 1985). When $\mathrm{N}$ fertility is high, nodule formation and $\mathrm{N}_{2}$ fixation of legumes is inhibited (Heichel, 1985), and grasses compete more successfully with legumes (Schwinning and Parsons, 1996). We found that $\mathrm{N}$ fertilizer did not affect emergence in 1998 or 1999 when swards were repeatedly cut at $5 \mathrm{~cm}$ before and after seeding. Nitrogen fertilizer, however, reduced legume emergence in $13-\mathrm{cm}$ and uncut swards. Overall legume survival was also affected less by $\mathrm{N}$ fertilization in $5-\mathrm{cm}$ swards than in $13-\mathrm{cm}$ and uncut swards.

Competition from grass is likely greater on summits than on backslopes because above-ground dry matter production of grass tends to be greater on summits than on backslopes (Harmoney et al., 2001). From 0 to 44 $\mathrm{kg} \mathrm{ha}{ }^{-1} \mathrm{~N}$, overall legume survival was on average greater on backslopes than on summits by 64 plants $\mathrm{m}^{-2}$ in 1998 and 105 plants $\mathrm{m}^{-2}$ in 1999 . Addition of $89 \mathrm{~kg}$ $\mathrm{ha}^{-1} \mathrm{~N}$ was required to reduce overall legume survival on backslopes to similar densities of survival on summits (Fig. 1). Growth rate of the sward appears to be greater on summits compared with backslopes. Leaf area index of the sward was initially greater and increased at a greater rate on summits than on backslopes from 28 May 1998 to 22 June 1998 and from 22 June 1999 to 16 July 1999.

Though we did not conduct this study across multiple locations or hillslopes, we expect competition from grasses to be greater on summits and overall legume survival to be greater on backslopes among other pastures typical of southeastern Iowa, and that these results would occur regardless of aspect. Aspect did not affect legume abundance or the production of grass dry matter in a previous experiment conducted in southeastern Iowa pastures (Harmoney et al., 2001).

Red clover had the greatest emergence and survival of the three legumes when sown together at similar rates in these cool-season grass pastures. Red clover was the predominant legume regardless of landscape position, $\mathrm{N}$ fertilizer rate, or sward height. Alfalfa and birdsfoot trefoil contributed significantly to overall legume survival when swards were cut at $5 \mathrm{~cm}$ and $\mathrm{N}$ fertilizer rates were 0 to $22 \mathrm{~kg} \mathrm{ha}^{-1}$. In sown mixtures, the diversity of seedlings that establish may be lower in $\mathrm{N}$-fertilized grasslands because accumulated litter and lower light penetration inhibits germination and/or survival of seedlings (Tilman, 1993). Previous research has shown that red clover is affected less by low availability of light than alfalfa and birdsfoot trefoil (Gist and Mott, 1957). It remains unknown, however, as to how emergence and survival of these legumes might be affected were they to be sown as a mixture in pastures with different limiting conditions.

Our study shows that legume establishment is limited by seedling survival on summit positions in pastures. Establishment of legumes is successful on backslopes because of less competition from grass. Increased sward height and $\mathrm{N}$ fertility enhance grass competitiveness, thus reducing both emergence and survival of legumes in pastures. We recommend legumes be seeded on backslope landscape positions in pastures and that $\mathrm{N}$ fertilizer not be applied, and sward heights be reduced before seeding.

\section{REFERENCES}

Ballaré, C.L., A.L. Scopel, and R.A. Sánchez. 1990. Far-red radiation reflected from adjacent leaves: An early signal of competition in plant communities. Science (Washington, DC) 24:329-332.

Cuomo, G.J., D.G. Johnson, and W.A. Head, Jr. 2001. Interseeding kura clover and birdsfoot trefoil into existing cool-season grass pastures. Agron. J. 93:458-462.

Gist, G.R., and G.O. Mott. 1957. Some effects of light intensity, temperature, and soil moisture on the growth of alfalfa, red clover, and birdsfoot trefoil seedlings. Agron. J. 49:33-36.

Groya, F.L., and C.C. Sheaffer. 1981. Establishment of sod-seeded alfalfa at various levels of soil moisture and grass competition. Agron. J. 73:560-565.

Harmoney, K.R., K.J. Moore, E.C. Brummer, C.L. Burras, and J.R. George. 2001. Spatial legume composition and diversity across seeded landscapes. Agron. J. 93:992-1000.

Heichel, G.H. 1985. Symbiosis: Nodule bacteria and leguminous plants. p. 64-71. In D.S. Metcalfe (ed.) Forages: The science of grassland agriculture. 4th ed. Iowa State Univ. Press, Ames.

Heichel, G.H., and K.I. Henjum. 1991. Dinitrogen fixation, N transfer, and productivity of forage legume-grass communities. Crop Sci. 31:202-208.

McIntosh, M.S. 1983. Analysis of combined experiments. Agron. J. 75:153-155.

Schwinning, S., and A.J. Parsons. 1996. Analysis of the coexistence mechanisms for grasses and legumes in grazing systems. J. Ecol. 84:799-813.

Sleugh, B., K.J. Moore, J.R. George, and E.C. Brummer. 2000. Binary legume-grass mixtures improve forage yield, quality, and seasonal distribution. Agron. J. 92:24-29.

Taylor, R.W., and D.W. Allinson. 1983. Legume establishment in grass sods using minimum-tillage seeding techniques without herbicide application: Forage yield and quality. Agron. J. 75:167-172.

Tilman, D. 1993. Species richness of experimental productivity gradients: How important is colonization limitation? Ecology 74:21792191.

Trott, J.O., K.J. Moore, V.L. Lechtenberg, and K.D. Johnson. 1988. Light penetration through tall fescue in relation to canopy biomass. J. Prod. Agric. 1:137-140.

Van Soest, P.J. 1982. Nutritional ecology of the ruminant. O \& B Books, Corvallis, OR. 